\section{Refining sorafenib therapy: lessons from clinical practice}

\author{
Luigi Bolondi ${ }^{* 1}$, Antonio Craxi ${ }^{2}$, Franco Trevisani ${ }^{3}$, Bruno Daniele ${ }^{4}$, Giovan Giuseppe \\ Di Costanzo ${ }^{5}$, Stefano Fagiuoli ${ }^{6}$, Calogero Cammà7, Paolo Bruzzi ${ }^{8}$, Romano Danesi ${ }^{9}$, \\ Federico Spandonaro ${ }^{10}$, Corrado Boni ${ }^{11}$, Armando Santoro ${ }^{12} \&$ Massimo Colombo ${ }^{13}$
}

\begin{abstract}
Understanding the best use of sorafenib is essential in order to maximize clinical benefit in hepatocellular carcinoma. Based on Phase III and noninterventional study data, as well as our extensive experience, we discuss dose modification in order to manage adverse events, disease response evaluation and how to maximize treatment benefit. Sorafenib should be initiated at the approved dose (400 mg twice daily) and reduced/interrupted as appropriate in order to manage adverse events. Dose modification should be considered before discontinuation. Appropriate tumor response assessment is critical. Focusing on radiologic response may result in premature sorafenib discontinuation; symptomatic progression should also be considered. If second-line therapies or trials are unavailable, continuing sorafenib beyond radiologic progression may provide a clinical benefit. Our recommendations enable the maximization of treatment duration, and hence clinical benefit, for patients.
\end{abstract}

It is well established that hepatocellular carcinoma (HCC) is a complex and heterogeneous disease that is affected by multiple genetic and epigenetic alterations [1]. In the overwhelming majority of patients, liver cirrhosis - another complex disease in its own right - is superimposed on HCC. As a result, the prognostic range for these patients varies considerably and continues to change for each patient at every time point over the clinical course of their disease. A meta-analysis of 30 clinical trials into which HCC patients were enrolled for palliative treatment reflects this heterogeneity. It demonstrated a high variability in survival among untreated patients and concluded that no single patient characteristic alone could predict outcome [2].

The prognosis and treatment options for HCC are generally related to tumor stage and liver function at presentation [3,4]. In the west, approximately $30 \%$ of all patients with HCC are diagnosed in

'Division of Internal Medicine, Department of Medical \& Surgical Sciences, University of Bologna, S Orsola-Malpighi Hospital, Bologna, Italy 2Dipartimento Biomedico di Medicina Interna e Specialistica \& UOC Gastroenterologia \& Epatologia, Di.Bi.M.I.S. Università di Palermo,

Palermo, Italy

${ }^{3}$ Dipartimento di Scienze Mediche Chirurgiche, Unità di Semeiotica Medica, Alma Mater Studiorum - Università di Bologna, Bologna, Italy

${ }^{4}$ Department of Oncology, G Rummo Hospital, Benevento, Italy

sLiver Unit, Cardarelli Hospital, Naples, Italy

'USC Gastroenterologia ed Epatologia dei Trapianti Dipartimento di Medicina Specialistica e dei Trapianti Azienda Ospedaliera Papa

Giovanni XXIII, Bergamo, Italy

7 Section of Gastroenterology, DIBIMIS, University of Palermo, Palermo, Italy

${ }^{8}$ Clinical Epidemiology, IRCCS AUO San Martino-IST Genoa, Genoa, Italy

${ }^{9}$ Dipartimento di Medicina Clinica e Sperimentale Università di Pisa, Pisa, Italy

${ }^{10}$ Università di Roma Tor Vergata, Rome, Italy

"Corrado Boni Arcispedale S Maria Nuova IRCCS, Reggio Emilia, Italy

${ }^{12}$ Humanitas Cancer Center, Istituto Clinico Humanitas IRCCS Rozzano, Milan, Italy

13First Division of Gastroenterology, Fondazione IRCCS Ca' Granda Maggiore Hospital, University of Milan, Milan, Italy

*Author for correspondence: Tel.: +3951 6362 260; Fax: +39516362 725; luigi.bolondi@unibo.it

\section{KEYWORDS}

- adverse event

management

- Child-Pugh B • dose

modification • elderly

- hepatocellular

carcinoma • mRECIST

- postprogression treatment

- real-world data • response

assessment • sorafenib 
the early stages of the disease. For these patients, potentially curative treatment options, such as resection, transplantation and radiofrequency ablation, are available and offer a high probability of complete response. Approximately $20 \%$ of patients diagnosed with HCC have intermediate-stage disease and they can gain survival benefits of, on average, 20 months from treatment with transarterial chemoembolization (TACE), a locoregional treatment. However, for patients in the advanced stages of disease, who account for the majority of the HCC population, their disease is beyond resection and locoregional treatments are deemed ineffective. Consequently, the prognosis for advanced-stage $\mathrm{HCC}$ is poor [4].

Historically, the treatment options for advanced HCC were limited until the approval of sorafenib by the US FDA and the EMA in 2007. Sorafenib remains the only systemic therapy to date to have demonstrated a survival benefit in advanced HCC $[5,6]$. Since approval was granted to sorafenib, physicians have accrued a wealth of experience with the fine-tuning of sorafenib in their daily clinical practice, and several 'real-world' studies have continued to investigate the safety and efficacy of sorafenib. Among them is a multinational postmarketing study, GIDEON, and the Italian field-practice study by the Sorafenib Italian Assessment (SOFIA) study group [7-11], as well as a number of other studies in Europe and North America [12-15]. These real-world experiences have allowed us to assess sorafenib in patients who are not selected by strict clinical trial criteria but by physician judgment, including patients with comorbidities and those receiving concomitant medication.

Owing to the underlying liver disease, HCC is a notoriously difficult cancer to treat. This is reflected in the lack of agents that were approved before sorafenib and in the failure of recent clinical trials of other systemic therapies to meet their primary end points [4,16-19]. As sorafenib is currently the only approved systemic therapy that is available for HCC, it is important for physicians to know how best to use this agent in clinical practice in order to maximize the therapeutic benefits for their patients.

With this in mind, a panel of Italian experts convened in Italy in April 2013, at a meeting funded by Bayer Italy, in order to discuss which treatment strategies for sorafenib may facilitate the greatest patient benefit in clinical practice.
The key questions addressed during the expert meeting focused on the following areas:

- The dosing of sorafenib: in particular, the starting dose, the mean dose given in clinical studies and the use of dose modifications (including reductions and temporary interruptions);

- The evaluation of treatment response: specifically, how to evaluate progression, whether clinical or radiologic progression should be used, which evaluation criteria should theoretically and can practically be applied and how to weigh up the relative importance of tumor versus cirrhosis progression;

- Adherence to clinical guidelines in daily practice: specifically, the extent to and way in which guidelines are applied and the time point when treatment with a systemic agent should be initiated;

- The duration of treatment (DoT): in particular, when to stop sorafenib treatment and which treatment to use beyond progression, considering a rechallenge with sorafenib or switching to a second-line therapy;

- Communication with the patient: in particular, effective adverse event (AE) management and motivating the patient in order to extend patient adherence to sorafenib treatment.

In this article, we provide a synopsis of the main conclusions from the expert panel meeting in the anticipation that this will provide practicing physicians and other stakeholders with an understanding of how real-world experiences with sorafenib can help us to further refine and improve the management of patients with HCC.

\section{The importance of starting right: sorafenib should be initiated at the approved dose}

A Phase I pharmacokinetic study of sorafenib in patients with solid tumors identified $800 \mathrm{mg} /$ day (administered as $400 \mathrm{mg}$ twice daily) as the optimal dose to be tested in Phase II and III clinical trials of HCC [5-6,20-21]. This Phase I study was conducted in noncirrhotic patients. A subsequent Phase II trial using the same dose of sorafenib in cirrhotic Child-Pugh A and $\mathrm{B}$ patients with HCC detected no significant differences in the plasma pharmacokinetics of sorafenib between the two Child-Pugh groups (according to area under the curve, $\mathrm{C}_{\max }$ and 
Table 1. Key outcomes from the SHARP ${ }^{\dagger}$ and Asia-Pacific ${ }^{\ddagger}$ randomized, placebo-controlled, Phase III trials of sorafenib in advanced hepatocellular carcinoma: selected baseline characteristics and efficacy data.

\begin{tabular}{|c|c|c|c|c|c|c|c|c|}
\hline \multirow[t]{2}{*}{ Characteristic/efficacy data } & \multicolumn{4}{|c|}{ SHARP trial $(n=602)$} & \multicolumn{4}{|c|}{ Asia-Pacific trial $(n=226)$} \\
\hline & $\begin{array}{l}\text { Sorafenib } \\
(n=299)\end{array}$ & $\begin{array}{l}\text { Placebo } \\
(n=303)\end{array}$ & $H R$ & $p$-value & $\begin{array}{l}\text { Sorafenib } \\
(n=150)\end{array}$ & $\begin{array}{l}\text { Placebo } \\
(n=76)\end{array}$ & $H R$ & $p$-value \\
\hline BCLC stage C (\%) & 82 & 83 & - & - & 95 & 96 & - & - \\
\hline \multicolumn{9}{|l|}{ Baseline liver cirrhosis } \\
\hline Child -Pugh A (\%) & 95 & 98 & - & - & 97 & 97 & - & - \\
\hline \multicolumn{9}{|l|}{ Response } \\
\hline Complete (\%) & 0 & 0 & - & - & 0 & 0 & - & - \\
\hline Partial (\%) & 2 & 1 & - & 0.05 & 3 & 1 & - & - \\
\hline Stable disease (\%) & 71 & 67 & - & 0.17 & 54 & 28 & - & - \\
\hline Disease control rate (\%) & 43 & 32 & - & 0.002 & 35 & 16 & - & 0.0019 \\
\hline Median radiologic TTP, months $(95 \% \mathrm{Cl})$ & $5.5(4.1-6.9)$ & $2.8(2.7-3.9)$ & 0.58 & $<0.001$ & $2.8(2.63-3.58)$ & $1.4(1.35-1.55)$ & 0.57 & 0.0005 \\
\hline Median symptomatic TTP, months ( $95 \% \mathrm{Cl})$ & $4.1(3.5-4.8)$ & $4.9(4.2-6.3)$ & 1.08 & 0.77 & $3.5(2.80-4.24)$ & $3.4(2.40-4.08)$ & 0.90 & 0.50 \\
\hline
\end{tabular}

$\mathrm{t}_{\max }$ values), confirming the tolerability of the 800 -mg dose in cirrhotic patients [20].

The approval of sorafenib for the treatment of HCC at a dose of $800 \mathrm{mg} /$ day was based on level I evidence from the SHARP trial, a randomized, Phase III trial in a western population [6], and was confirmed by another Phase III trial of similar design conducted in the Asia-Pacific region [5]. Tables 1 \& 2 summarize the main efficacy, safety and DoT data, as well as the rates of sorafenib dose modifications and discontinuations reported in these two trials. Since their publication, further support for initiating sorafenib treatment at the $800 \mathrm{mg} /$ day dose has emerged from clinical practice and been captured by observational studies, such as GIDEON and SOFIA, which reported similar median survival outcomes and toxicity profiles that were analogous to the SHARP trial. Tables $3 \& 4$ provide an overview of the study designs and key outcomes of these real-world studies.

Based on the strength of the trial data sorafenib is supported by the highest level of evidence among treatments for HCC (Figure 1) - sorafenib $800 \mathrm{mg} / \mathrm{day}$ is the recommended standard of care for patients with advanced HCC. It is also recommended for patients with intermediate HCC who are unsuitable for treatment with TACE or have TACE-refractory disease $[4,24]$

To our knowledge, no clinical trial in HCC has prospectively compared a different starting dose of sorafenib with the approved dose of $800 \mathrm{mg} / \mathrm{day}$. The SOFIA study reported outcomes for patients who maintained an initial dose of $800 \mathrm{mg} / \mathrm{day}$ and for patients in whom the initial dose was reduced in order to manage AEs [9]. In a retrospective analysis, the authors reported a median overall survival (OS) of 21.6 months for patients who received $400 \mathrm{mg} /$ day of sorafenib for $70 \%$ of the treatment period and 9.6 months for those who maintained full dosing or had a dose reduction for $<70 \%$ of the treatment period. These results are affected by an inherent selection bias, as longer survival may be the cause, rather than the consequence, of dose reductions - the longer a patient receives active treatment, the greater the opportunity for AEs to develop, which in turn may require dose reductions. Furthermore, certain side effects, such as hand-foot skin reaction (HFSR), may be pharmacodynamic indicators of an individual's susceptibility to sorafenib, and may thus be associated with better outcomes, although the underlying mechanisms are not yet understood [25-27]. Thus, the patients in the reduced-dose 
SPECIAL REPORT Bolondi, Craxi, Trevisani et al.

Table 2. Safety, dose reduction, treatment discontinuation and duration of treatment outcomes for the SHARP and Asia-Pacific trials. ${ }^{\dagger}$

\begin{tabular}{|c|c|c|c|c|c|c|c|c|}
\hline \multirow[t]{2}{*}{ Outcome } & \multicolumn{4}{|c|}{ SHARP trial $(n=602)$} & \multicolumn{4}{|c|}{ Asia-Pacific trial $(n=226)$} \\
\hline & \multicolumn{2}{|c|}{ Sorafenib $(n=299)$} & \multicolumn{2}{|c|}{ Placebo $(n=303)$} & \multicolumn{2}{|c|}{ Sorafenib $(n=150)$} & \multicolumn{2}{|c|}{ Placebo $(n=76)$} \\
\hline \multicolumn{9}{|l|}{ Treatment-emergent $A E^{\ddagger}$} \\
\hline All (\%) & \multicolumn{2}{|l|}{98} & \multicolumn{2}{|l|}{96} & \multicolumn{2}{|l|}{98} & \multicolumn{2}{|l|}{95} \\
\hline Serious (\%) & \multicolumn{2}{|l|}{52} & \multicolumn{2}{|l|}{54} & \multicolumn{2}{|l|}{48} & \multicolumn{2}{|l|}{45} \\
\hline \multicolumn{9}{|l|}{ Drug-related $A E$} \\
\hline All (\%) & \multicolumn{2}{|l|}{80} & \multicolumn{2}{|l|}{52} & \multicolumn{2}{|l|}{82} & \multicolumn{2}{|l|}{39} \\
\hline By severity grade & Any grade & Grade 3/4 & Any grade & Grade 3/4 & Any grade & Grade 3/4 & Any grade & Grade 3/4 \\
\hline HFSR (\%) & 21 & 8 & 3 & $<1$ & 45 & 11 & 3 & 0 \\
\hline Diarrhea (\%) & 39 & 8 & 11 & 2 & 26 & 6 & 5 & 0 \\
\hline Alopecia (\%) & 14 & 0 & 2 & 0 & 25 & 0 & 1 & 0 \\
\hline Fatigue (\%) & 22 & 4 & 16 & $<4$ & 20 & 3 & 8 & 1 \\
\hline Rash/desquamation (\%) & 16 & 1 & 11 & 0 & 20 & 1 & 7 & 0 \\
\hline Hypertension (\%) & 5 & 2 & 2 & 1 & 19 & 2 & 1 & 0 \\
\hline Anorexia (\%) & 14 & $<1$ & 3 & 1 & 13 & 0 & 3 & 0 \\
\hline Nausea (\%) & 11 & $<1$ & 8 & 1 & 11 & 1 & 11 & 1 \\
\hline \multicolumn{9}{|l|}{ Dose reduction } \\
\hline All (\%) & \multicolumn{2}{|l|}{26} & \multicolumn{2}{|l|}{7} & \multicolumn{2}{|l|}{31} & \multicolumn{2}{|l|}{3} \\
\hline HFSR (\%)" & \multicolumn{2}{|l|}{5} & \multicolumn{2}{|l|}{-} & \multicolumn{2}{|l|}{11} & \multicolumn{2}{|l|}{0} \\
\hline Diarrhea (\%)" & 8 & & - & & 7 & & 0 & \\
\hline Discontinuation & & & & & & & & \\
\hline All (\%) & 38 & & 37 & & 20 & & 13 & \\
\hline Hemorrhage, upper GI (\%) & 6 & & - & & 3 & & 4 & \\
\hline Ascites (\%) & - & & - & & 3 & & 3 & \\
\hline Fatigue (\%) & 5 & & - & & 3 & & 0 & \\
\hline Liver dysfunction (\%)" & 5 & & - & & $<1$ & & 3 & \\
\hline Dot & & & & & & & & \\
\hline Median DoT, months (range) & $5.3(0.2-16$. & & $4.3(0.1-16.6$ & & - & & - & \\
\hline $\begin{array}{l}\text { †Data taken from [5,6]. } \\
\text { "AE occurring in at least } 5 \% \text { of patien } \\
{ }^{5} \text { According to CTCAE } 33.0 \text {. } \\
\text { "Most frequent reasons for dose red } \\
\text { "Most frequent reasons for treatmen } \\
\text { AE: Adverse event; CTCAE: Common } \\
\text { skin reaction. }\end{array}$ & or Adverse Ev & Jents; DoT: Dur & on of treatme & ent; Gl: Gastrc & tinal; HCC: & tecellutax & nor & nd-foot \\
\hline
\end{tabular}

group may be the patients who most readily respond to the Raf-inhibitory (therapeutic) effects of sorafenib [25,27]. At the same time, on the same dose, patients in whom the pharmacokinetics of sorafenib are shifted towards the lower end of susceptibility or who have a more aggressive form of HCC will have a worse outcome. Hypothetically, in these patients, the paradoxical growth-enhancing effect of sorafenib at lower doses (reported in a rodent model) may also come into play $[28,29]$.

In this context, it is insightful to consider a pharmacokinetic study of sorafenib by Miller and colleagues in solid-cancer patients with hepatic or renal dysfunction [30]. Despite corroborating the results of another trial by failing to show a significant difference in the pharmacokinetics of a 400-mg dose of sorafenib between Child-Pugh A and B patients [31], the authors did find that higher bilirubin concentrations were associated with lower areas under the curve of the main sorafenib metabolite, $N$-oxidesorafenib, but only in the hepatic and not the renal cohort. Sorafenib was also only found to be associated with a dose-limiting elevation of bilirubin in patients with hepatic but not renal dysfunction. Miller et al. speculate that this intolerance may be linked to the inhibition of uridine diphosphate-glucuronosyl-transferase by sorafenib. 
Table 3. Overview of the study design and outcomes from the GIDEON and SOFIA real-world studies of sorafenib in HCC': study design and efficacy/effectiveness outcomes.

\begin{tabular}{|c|c|c|}
\hline Design/outcome & GIDEON trial $(n=3202)$ & SOFIA trial $(n=296)$ \\
\hline Study design & $\begin{array}{l}\text { Global, prospective, noninterventional study of patients } \\
\text { with unresectable HCC eligible for systemic therapy } \\
\text { and treated with sorafenib under real-life practice } \\
\text { conditions in order to evaluate the safety and efficacy of } \\
\text { sorafenib in different subgroups. The recruitment aim } \\
\text { was } 3000 \text { patients from }>40 \text { countries, with a follow-up } \\
\text { of approximately } 5 \text { years }\end{array}$ & $\begin{array}{l}\text { Italian, six-center, investigator-driven, } \\
\text { observational, noninterventional study in order to } \\
\text { assess the safety and effectiveness of sorafenib in } \\
\text { patients with advanced HCC or intermediate HCC } \\
\text { not eligible for or having failed ablative therapies. } \\
\text { Consecutive evaluation of patients took place } \\
\text { between } 2008 \text { and } 2012\end{array}$ \\
\hline \multicolumn{3}{|l|}{ Baseline HCC stage } \\
\hline BCLC stage B (\%) & 20 & 25 \\
\hline BCLC stage C (\%) & 52 & 75 \\
\hline \multicolumn{3}{|l|}{ Baseline liver cirrhosis } \\
\hline Child-Pugh A (\%) & 62 & 88 \\
\hline Child-Pugh B (\%) & 21 & 37 \\
\hline \multicolumn{3}{|l|}{ Median OS } \\
\hline Total (months) & - & 10.5 \\
\hline Child-Pugh A (months) & 13.6 & - \\
\hline Child-Pugh B (months) & 5.2 & - \\
\hline BCLC stage B (months) & - & 20.6 \\
\hline BCLC stage C (months) & - & 8.4 \\
\hline \multicolumn{3}{|l|}{ Median radiologic TTP } \\
\hline Overall (months) & - & 9.2 \\
\hline Child-Pugh A (months) & 4.7 & - \\
\hline Child-Pugh B (months) & 4.4 & - \\
\hline
\end{tabular}

Even in the real-world SOFIA study, the sorafenib starting dose was $800 \mathrm{mg} / \mathrm{day}$ and was reduced only as required by intolerance or AEs. Dose adjustments for the purpose of managing AEs are a valid strategy for avoiding permanent discontinuation where possible and thus ensuring that no treatment benefit is lost.

Real-world noninterventional studies, such as GIDEON, have so far not delivered evidence to suggest that a lower starting dose of sorafenib may improve or at least result in equal clinical effectiveness compared with the recommended starting dose of $800 \mathrm{mg} /$ day $[25,32]$. Data from GIDEON showed that patients on the higher, approved, 800-mg starting dose had better efficacy outcomes than patients starting treatment on a dose of $400 \mathrm{mg} /$ day [11,22]. In this real-world study, $74 \%$ of patients started sorafenib treatment at the approved dose. In terms of HCC disease stage, liver function and prior surgery or locoregional treatment, these patients were similar to the $22 \%$ of patients given the $400 \mathrm{mg} /$ day starting dose. On average, patients who started treatment at $800 \mathrm{mg} /$ day remained on treatment for 2.6 weeks longer than patients starting on $400 \mathrm{mg} /$ day. In these patients, median OS was extended by over 2 months and median time to progression (TTP) was extended by approximately 1 month compared with the lower-starting-dose group (9.3 vs 7.1 months and 4.5 vs 3.6 months, respectively) [22]. In terms of safety, the findings for the approved- and low-dose groups were similar, with approximately $30 \%$ of patients receiving sorafenib dose modifications irrespective of the starting dose $(35 \%$ with $800 \mathrm{mg} /$ day and $32 \%$ with $400 \mathrm{mg} /$ day) and no significant differences in the types or incidences of AEs between the two groups [11,22]. A recent subanalysis of European patients included in the GIDEON noninterventional study has closely echoed these findings [23].

To stop or not to stop sorafenib? When is the question

The median DoT with sorafenib was 5.3 months in the Phase III SHARP trial and 3.8 and 3.4 months in the real-world SOFIA and GIDEON studies, respectively [6,9-10], suggesting that sorafenib may be stopped prematurely 
in some clinical scenarios. It is crucial to understand the key drivers behind why this may happen. Two possible explanations include discontinuation because of sorafenib-associated or disease-related AEs and/or a perceived absence of tumor response and clinical benefit with sorafenib.
- How AEs may influence treatment decisions with sorafenib

Understanding how to optimize the use of sorafenib in daily clinical practice - including how to modify the dose appropriately according to the type and severity of AEs and how to effectively manage sorafenib-associated AEs - is important

Table 4. Safety, dose reduction, treatment discontinuation and duration of treatment outcomes for the GIDEON and SOFIA trials.

\begin{tabular}{|c|c|c|c|c|}
\hline \multirow{2}{*}{$\begin{array}{l}\text { Outcome } \\
\text { Treatment-emergent } A E^{\dagger}\end{array}$} & \multicolumn{2}{|c|}{ GIDEON trial $(n=3202)$} & \multicolumn{2}{|c|}{ SOFIA trial $(n=296)$} \\
\hline & Any grade & Grade 3/4 & Any grade & Grade 3/4 \\
\hline Overall (\%) & 85 & 30 & 91 & 45 \\
\hline Serious AE (\%) & 43 & - & - & - \\
\hline Drug-related $A E$ & Any grade & Grade $3 / 4$ & Any grade & Grade $3 / 4$ \\
\hline Overall (\%) & 66 & 23 & - & - \\
\hline Drug-related serious AE (\%) & 9 & - & - & - \\
\hline HFSR (\%) & 27 & - & 28 & 9 \\
\hline Diarrhea (\%) & 27 & - & 35 & 6 \\
\hline Alopecia (\%) & 7 & - & - & - \\
\hline Fatigue (\%) & 16 & - & 66 & 25 \\
\hline Rash/desquamation (\%) & 11 & - & 5 & 2 \\
\hline Hypertension (\%) & 9 & - & 18 & 7 \\
\hline Anorexia (\%) & 10 & - & - & - \\
\hline Nausea (\%) & 7 & - & 11 & 3 \\
\hline Weight loss (\%) & - & - & 39 & 6 \\
\hline Constipation (\%) & - & - & 16 & 0 \\
\hline Stomatitis (\%) & - & - & 6 & 0 \\
\hline GI bleeding (\%) & - & - & 9 & 5 \\
\hline Any cardiovascular event (\%) & - & - & 5 & 2 \\
\hline \multicolumn{5}{|l|}{ Dose reduction } \\
\hline Overall (\%) & $33^{\ddagger}$ & & 54 & \\
\hline Any $\mathrm{AE}(\%)^{\S}$ & - & & 83 & \\
\hline Deteriorated liver function $(\%)^{\S}$ & - & & 9 & \\
\hline Fatigue $(\%)^{\S}$ & - & & 21 & \\
\hline HFSR $(\%)^{\S}$ & - & & 10 & \\
\hline Diarrhea $(\%)^{\S}$ & - & & 8 & \\
\hline \multicolumn{5}{|l|}{ Discontinuation } \\
\hline Overall (\%) & - & & 79 & \\
\hline Any $A E(\%)^{\#}$ & 31 & & 32 & \\
\hline Fatigue (\%) & - & & 7 & \\
\hline Deteriorated liver function (\%) & - & & 13 & \\
\hline HCC progression (\%)\# & - & & 34 & \\
\hline \multicolumn{5}{|l|}{ Median DoT } \\
\hline Overall (months) & 3.5 & & 3.8 & \\
\hline If interrupted due to $A E$ (months) & - & & 1.7 & \\
\hline If interrupted due to progression (months) & - & & 8.7 & \\
\hline \multicolumn{5}{|l|}{$\begin{array}{l}{ }^{\dagger} \mathrm{AE} \text { occurring in at least } 5 \% \text { of patients. } \\
\ddagger 2 \text { nd interim analysis, } N=1571 \text {. } \\
\text { \$Most frequent reasons for dose reduction. } \\
\text { "Most frequent reasons for treatment discontinuation. } \\
\text { AE: Adverse event; DoT: Duration of treatment; HCC: Hep } \\
\text { specified. }\end{array}$} \\
\hline
\end{tabular}




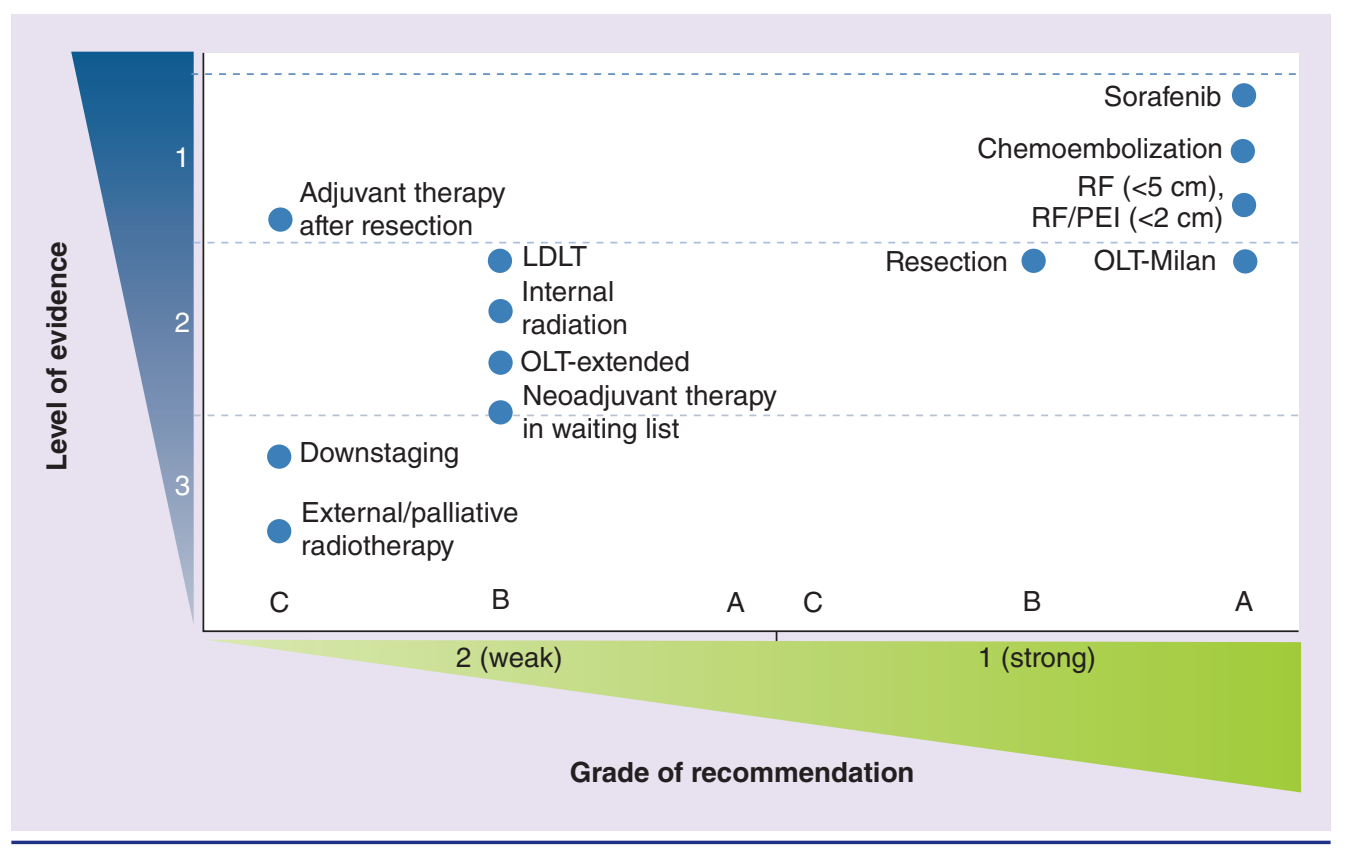

Figure 1. Sorafenib is associated with the highest level of evidence in the European Association for the Study of the Liver-European Organisation for Research and Treatment of Cancer guidelines for the management of hepatocellular carcinoma.

LDLT: Living-donor liver transplantation; OLT: Orthotopic liver transplantation; PEI: Percutaneous ethanol injection; RF: Radiofrequency ablation.

Reproduced with permission from [4] @ (2012) European Association for the Study of the Liver.

in order to achieve the longest possible DoT, and with this, the best possible patient benefit.

\section{Sorafenib dose modification during therapy}

is a viable treatment strategy for maximizing patient outcomes

Real-world studies of patients with HCC have confirmed that sorafenib has an AE profile that is consistent with the those observed in Phase III clinical trials, offering reassurance that the management strategies already developed in the clinical trial setting remain applicable in the real-world setting [5-6,9-11,22-23]. In advanced HCC, AEs due to sorafenib treatment occur mainly during the first month of treatment and progressively reduce in frequency thereafter [13]. It is therefore imperative that patients should be monitored closely, especially during the first weeks of therapy.

As observations from the SOFIA study have shown, if patients with sorafenib-associated AEs are managed appropriately by adopting appropriate dose modification or treatment interruption strategies promptly, they can be maintained on treatment for longer, resulting in improved clinical outcomes [9]. All patients in the SOFIA study were started on the recommended full dose of sorafenib: $800 \mathrm{mg} /$ day. In the event of clinically relevant grade 3 or 4 AEs, the sorafenib dose was reduced to $400 \mathrm{mg} /$ day or a temporary dose interruption was introduced until symptoms resolved to grade 1 or 2 severity, in accordance with the sorafenib label. Re-escalation to the full dose was performed when possible. Deterioration of hepatic function due to the underlying liver disease was another criterion for dose modification or interruption. Revealingly, the median DoT in the $26 \%$ patients who had undergone dose modification in order to manage AEs for more than $70 \%$ of the time was longer ( 6.8 months) than in patients who had been maintained on the recommended dose $(800 \mathrm{mg} /$ day) for more than $70 \%$ of the time (3 months); the patients with dose modifications had a median OS of 21.6 months. Thus, in patients with longer survival times, dose reductions were frequently reported, suggesting that these reductions do not abolish the beneficial effects of sorafenib (see Table 4 for an overview of the dose reduction and discontinuation findings of the SOFIA study).

In addition, reports of individual case studies have highlighted the importance of prolonged sorafenib administration, even at a reduced dose. Abbadessa and colleagues observed two partial and two complete responses, with progressionfree survival ranging from 12 to 62 months, after 
Table 5. Summary of dose modifications for skin toxicity.

\section{Skin toxicity grade}

Grade 1: numbness, dysesthesia, paresthesia,

tingling, painless swelling, erythema or

discomfort of the hands or feet that does not

disrupt the patient's normal activities

Grade 2: painful erythema and swelling of the

hands or feet and/or discomfort affecting the

patient's normal activities

Grade 3: moist desquamation, ulceration,

blistering or severe pain of the hands or feet or

severe discomfort that causes the patient to be

unable to work or perform activities of daily living Third occurrence

Reproduced from [3] with permission of the European Society for Medical Oncology.

Any occurrence

First occurrence occurrence

First or second

\section{Suggested dose modification}

Continue treatment with sorafenib and consider topical therapy for symptomatic relief

Continue treatment with sorafenib and consider topical therapy for symptomatic relief. If no improvement within 7 days, see below

No improvement within 7 days or second or third Interrupt sorafenib treatment until toxicity resolves to grade $0-1$. When resuming treatment, decrease sorafenib dose by one dose level (400 mg daily or $400 \mathrm{mg}$ every other day)

Fourth occurrence Discontinue sorafenib treatment Interrupt sorafenib treatment until toxicity resolves to grade $0-1$. When resuming treatment, decrease sorafenib dose by one dose level (400 mg daily or $400 \mathrm{mg}$ every other day) Discontinue sorafenib treatment maintaining four patients on sorafenib for over 12 months (in one case even 30 months) through the carefully judged use of dose-modification strategies [33]. At this juncture, it is appropriate to highlight that the DoT in the clinical practice setting may be inherently shorter in some patients in which the $800 \mathrm{mg} /$ day dose is not reduced (e.g., if sorafenib is stopped earlier in patients with a more aggressive disease [and therefore with a poorer prognosis] or when the pharmacokinetics variability of sorafenib results in reduced exposure and possible decreased efficacy in some individuals).

An example of how sorafenib dose modifications can be implemented effectively in clinical practice is illustrated by the guidance for HFSR management (Table 5) [3]. Whether triggered by HFSR or another AE, the dose-modification strategy should take into consideration the reescalation of sorafenib, after sufficient improvement of the AE. This step will ensure that the clinical benefit associated with sorafenib treatment is maximized.

In patients who tolerate the treatment less well, sorafenib dose modification should always be considered first before permanent discontinuation

Certain patient groups naturally elicit a more conservative approach when it comes to dosing with sorafenib [25]. One such group comprises the Child-Pugh B population, which has been reported to fare worse than Child-Pugh A patients. In the SOFIA study, Iavarone et al. observed the deterioration of hepatic function in a greater proportion of Child-Pugh B (40\%) than Child-Pugh A patients (16\%) after 24 weeks of sorafenib treatment [9]. The GIDEON study reported that the incidence of drug-related AEs was generally consistent across ChildPugh A and B subgroups, although serious AEs were more common in Child-Pugh B patients. This study also showed that discontinuation of treatment due to AEs in the real-world setting was more common in Child-Pugh B patients (40.1 vs 28.9\%) [5-6,9-11,22-23].

Elderly patients make up another group. A recent review of this population in HCC trials (in which they are usually chronically under-represented) suggests that the elderly are treated more conservatively compared with younger patients at the same stage of disease, but concludes that age cannot be defined a contraindication to therapy at present because it does not adversely affect outcome, although it may condition treatment allocation [34]. However, until the availability of data from pharmacokinetic/pharmacodynamic analyses of individual patients with different clinical characteristics, which may allow for a more personalized therapy approach (e.g., tailored to severe comorbidities or impaired performance status), expert opinion clearly recommends that early dose modifications should be explored in patients who are intolerant of the full dose of sorafenib before a decision is taken to suspend treatment entirely [32].

There is a broad range of treatment strategies available to effectively manage or prevent AEs with sorafenib

Advising patients on preventive strategies, emphasizing the importance of early detection and 
explaining the clinical relevance of AEs all play a crucial role in avoiding the premature discontinuation of sorafenib. To this end, a wide variety of recommendations have been developed by multidisciplinary teams and are available in order to guide physicians in their management of the most common AEs associated with sorafenib [35-41]. Recommendations for managing HFSR, diarrhea, fatigue, and hypertension are shown in Table 6 and Boxes 1-4.

\section{- Response criteria for targeted therapies: ensuring sorafenib is maintained in order to maximize clinical outcomes}

There is much debate as to which criteria should be used in order to assess tumor response to targeted therapies, such as sorafenib [47]. The appropriate assessment of tumor response is critical for ensuring that treatment is not discontinued prematurely because of a perceived absence of clinical benefit.

Traditional Response Evaluation Criteria In Solid Tumors may not be appropriate for measuring response to sorafenib

Treatment response to cytotoxic agents has typically been evaluated with the Response Evaluation Criteria In Solid Tumors (RECIST) [48]. However, after the advent of targeted cancer therapies, RECIST was found to be suboptimal because of its restriction to tumor shrinkage as the sole measure of response. As a consequence, RECIST may not accurately reflect responses to treatments that cause tumor necrosis without an initial shrinkage of the tumor dimensions, as occurs with locoregional therapies $[47,48]$. This has raised the question of whether physicians are stopping treatment with targeted therapies, including sorafenib, too soon as a consequence of not being able to detect or evaluate tumor response reliably. The modified RECIST (mRECIST) criteria were developed in order to accommodate the requirement for both tumor necrosis and viability assessment (Table 6) $[42,47]$ and are recommended by current treatment guidelines for evaluating tumor response in patients with HCC $[4,24]$. However, although preliminary retrospective evidence supports the use of $\mathrm{mRE}$ CIST in HCC in order to assess tumor response to sorafenib, additional validation in larger studies is required [25,49-50].

\section{Sorafenib should not be discontinued based on radiologic response alone}

By relying on radiologic response alone, physicians may be stopping sorafenib too early. It is important to note that both of the Phase III trials of sorafenib in advanced HCC, on the basis of which this treatment was approved, stipulated that sorafenib was to be continued until the occurrence of both radiologic (at that time using RECIST prior to the definition of $\mathrm{mRECIST)}$ and symptomatic progression [5-6,9]. Indeed, one of the defined primary outcomes in these trials was time to symptomatic progression using the Functional Assessment of Cancer Therapy - Hepatobiliary Symptom Index 8 (FHSI8) questionnaire in order to assess symptomatic progression [5,6]. The approach of using nonradiologic progression as an indicator of when to cease sorafenib treatment was also adopted by the SOFIA group in their field practice study, in which treatment was continued until either radiologic or symptomatic progression [9]. In order to extend OS and improve quality of life for the patient, it is thus paramount that other clinical factors and read-outs of oncologic effect

Table 6. Summary of the Response Evaluation Criteria In Solid Tumors and the modified Response Evaluation Criteria In Solid Tumors.

\section{RECIST}

CR: disappearance of all target lesions

PR: at least a $30 \%$ decrease in the sum of diameters of

target lesions, taking as reference the baseline sum of the

diameters of target lesions

SD: any cases that do not qualify for either PR or PD

PD: an increase of at least $20 \%$ in the sum of the diameters

of target lesions, taking as reference the smallest sum of the

diameters of target lesions recorded since treatment started

CR: Complete response; mRECIST: Modified response evaluation criteria in solid tumors; SD: Stable disease.

Reproduced with permission from [44] ๑ (2010) Georg Thieme Verlag KG.

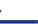

\section{mRECIST}

CR: disappearance of any intratumoral enhancement in all target lesions PR: at least a $30 \%$ decrease in the sum of diameters of viable (enhancement in the arterial phase) target lesions, taking as reference the baseline sum of the diameters of target lesions

SD: any cases that do not qualify for either PR or PD

PD: an increase of at least $20 \%$ in the sum of the diameters of viable (enhancing) target lesions, taking as reference the smallest sum of the diameters of viable (enhancing) target lesions recorded since treatment started id tumors; PD: Progressive disease; PR: Partial response; RECIST: Response evaluation criteria in solid 


\section{Box 1. Recommendations for the management of rash and hand-foot skin reaction with sorafenib treatment.}

- Inform patients of the full range of rash and HFSR symptoms so that they know what to expect

- Advise patients that prompt reporting and treatment of mild HFSR may prevent HFSR progression and allow continued full-dose therapy. Advise patient to keep good daily hygiene procedures

- Treat any pre-existing dermatologic conditions

- Specific recommendations for HFSR:

- Application of keratolytic creams (containing urea, $\alpha$-hydroxy acids or salicylic acid) in order to aid natural exfoliation; emollient creams for moisturization; appropriate use of topical corticosteroids; and topical analgesics

- Specific recommendations for rash:

- Management with over-the-counter body lotions containing exfoliative $\alpha$-hydroxy acid components, zinc oxide-based emollients, lanolin-based creams and antihistamines

- Dose adjustment to be used for severe cases of rash and HFSR, with re-escalation to full sorafenib dose if tolerated once AEs have resolved Rash and HFSR are among the most common dermatologic AEs associated with sorafenib.

AE: Adverse event; HFSR: Hand-foot skin reaction.

Recommendations are supported by $[3,35-38,41,60-61]$

are considered alongside radiologic response. According to clinical judgment, treatment with sorafenib should be maintained in patients who continue to demonstrate a clinical benefit from therapy (i.e., a stable clinical condition).

\section{Identifying markers of tumor response may help to guide sorafenib therapy \& predict prognosis}

The identification of biological markers is an exciting area of current translational research and holds the possibility of eventually tailoring treatment and/or dosing to best effect in individual patients. One objective of the SHARP trial was to explore the ability of plasma biomarkers to predict patient prognoses and sorafenib efficacy. The authors of the SHARP biomarker analysis reported that baseline angiopoietin 2 , VEGF concentrations, $\alpha$-fetoprotein and alkaline phosphatase concentrations, as well as macroscopic vascular invasion and Eastern
Cooperative Oncology Group (ECOG) status, independently predicted survival in the patient population as a whole, and that high s-c-KIT or low HGF concentrations at baseline were associated with a trend in favor of improved survival in patients treated with sorafenib [51]. However, no biomarker has yet been validated for selecting patients for sorafenib therapy.

Other recent studies have investigated plasma biomarkers as potential markers of response to sorafenib (and other antiangiogenic therapies), such as $\alpha$-fetoprotein [52-55], and additional research is ongoing. There are also some small studies that suggest that sorafenib AEs (e.g., skin toxicity and hypertension) may be markers of clinical efficacy and that the pattern of disease progression on sorafenib treatment may predict the postprogression prognosis [27,56]. This information may be useful in the design of second-line studies as well as in clinical decision-making for the postprogression continuation of sorafenib.

\section{Box 2. Recommendations for the management of diarrhea with sorafenib treatment.}

- Encourage patients to use a stool diary, report any abnormalities or deterioration in symptoms and seek medical advice if concerned

- Discuss dietary measures with the patient:

- Avoid high-fiber foods or those that aggravate diarrhea

- Implement dehydration prevention management:

- For example, by aggressive oral rehydration with electrolytes

- Consider pharmacologic management (e.g., loperamide):

- Loperamide schedule recommended by a European nurse task group [38]:

- $4 \mathrm{mg}$ followed by $2 \mathrm{mg}$ every $2 \mathrm{~h}$ until $2 \mathrm{~h}$ after the last bowel movement

- For patients with long-term diarrhea, 2-4 mg twice daily titrated according to bowel function

- A preventative measure in order to avoid recurrent diarrhea is to take loperamide $30 \mathrm{~min}$ prior to sorafenib treatment

- Dose adjustment to be used as necessary for grade 3-4 diarrhea (dose reduction and/or interruption), with re-escalation to full sorafenib dose if tolerated once the $\mathrm{AE}$ has resolved

Management of diarrhea should be carried out by a hepatologist, who can adequately consider complications potentially arising in cirrhotic patients. 


\section{Box 3. Recommendations for the management of fatigue with sorafenib treatment.}

- Advise the patient to self-monitor fatigue levels and adopt energy-conserving strategies when needed

- Consider nonpharmacological interventions:

- Activity enhancement (e.g., exercise as appropriate)

- Cognitive behavioral therapy

- Nutrition consultation

- Consider pharmacological interventions:

- Psychostimulants

- Treatment for pain, emotional distress, anemia and hypothyroidism

- Treatment for sleep dysfunction, nutritional imbalances/disturbances and comorbidities

- Dose adjustment

- Grade 3-4 fatigue requires treatment interruption or dose adjustment, with re-escalation to full sorafenib dose if tolerated once the AE has resolved

Fatigue is an underlying symptom of cancer and other comorbidities. Investigation of the possible reasons for fatigue is important in order to implement the appropriate management

AE: Adverse event.

Recommendations are supported by $[3,40,60,63]$.

However, data from large, prospective studies are required in order to confirm this hypothesis.

Finally, it has been shown that the pattern of tumor progression that is observed during sorafenib treatment may impact on survival and could potentially be used in order to determine patient prognosis [12]. For example, the appearance of new extrahepatic lesions is associated with worse postprogression survival than progression without new extrahepatic lesions (7.1 vs 14.9 months, respectively; $\mathrm{p}=0.02$ ).

\section{Using sorafenib in Child-Pugh B patients}

Child-Pugh B patients are of special interest in terms of sorafenib management, as the progression of their cirrhosis, rather than tumor progression, may result in the discontinuation of sorafenib. As a whole, the data available on the safety of sorafenib in Child-Pugh B patients suggest the feasibility of using this treatment in this population, taking into account the fact that poorer clinical outcomes are to be expected due to worse liver function [3]. However, more robust studies are necessary before confirming or disregarding the use of sorafenib in this subset of patients [3]. The ongoing BOOST Phase III study is aiming to address this question. This study will compare OS with sorafenib $(800 \mathrm{mg} /$ day) versus best supportive care in 320 patients with HCC and impaired liver function (Child-Pugh B; NCT01405573). In addition, the ongoing PRODIGE 21 Phase II randomized trial will evaluate time to radiologic progression in 160 patients with HCC and Child-Pugh B cirrhosis receiving sorafenib alone, pravastatin alone or the combination of sorafenib and pravastatin (NCT01357486).

\section{Box 4. Recommendations for the management of hypertension with sorafenib treatment.}

- Monitor blood pressure regularly:

- Once a week for the first 6 weeks of sorafenib treatment

- Continue weekly monitoring in patients with pre-existing hypertension

- Pre-existing hypertension:

- Mild-to-moderate increases in blood pressure can be managed by increasing the dose of the patient's current antihypertensive medication or adding a new antihypertensive medication

- New-onset hypertension:

- This can be effectively managed with standard antihypertensive therapy (appropriate to the individual patient and clinical situation), which includes angiotensin II receptor blockers and angiotensin-converting enzyme inhibitors, according to standard practice guidelines (e.g., European Society of Cardiology/European Society of Hypertension guidelines)

- Dose adjustment

- Dose reductions/interruptions can be used in order to manage hypertension if required, and in cases of severe or persistent hypertension that do not respond to antihypertensive drug treatment, permanent discontinuation of sorafenib should be considered

In patients receiving sorafenib, hypertension is usually mild to moderate, occurs early in therapy and can often be managed with standard antihypertensive therapy. AE: Adverse event.

Recommendations are supported by $[60,62]$ 
In the GIDEON study, $61.5 \%$ of patients were classified as having Child-Pugh A status and $20.8 \%$ as having Child-Pugh B status [10]. Overall, this noninterventional study showed that a greater proportion of Child-Pugh B than Child-Pugh A patients discontinued sorafenib because of AEs (40.1 vs $28.9 \%$ ) or experienced grade $3 / 4$ AEs (14.1 vs $8.8 \%$ ). Nevertheless, just over a quarter of patients with Child-Pugh B cirrhosis $(25.7 \%)$ were treated with sorafenib for over 24 weeks and the TTP was comparable between the Child-Pugh A and B cohorts (4.7 vs 4.4 months, respectively); however, not surprisingly, median survival in the Child-Pugh B cohort was shorter by approximately 8 months (13.6 vs 5.2 months; Table 3).

Similar findings were also observed in a multicenter, open-label, Phase II trial of sorafenib ( $800 \mathrm{mg} /$ day) performed in 297 patients with Child-Pugh A (79\%) and Child-Pugh B (21\%) liver function [14]. Compared with their ChildPugh A counterparts, patients with Child-Pugh B status had shorter progression-free survival (PFS; 2.1 vs 4.3 months), moderately shorter TTP (3.8 vs 4.2 months) and clearly reduced OS (3.8 vs 10.0 months). Again, the overall AE profile was similar in the two groups.

The results of these studies are not surprising, given that patients with Child-Pugh B liver function have a poorer prognosis as a consequence of their more advanced liver cirrhosis, justifying the development of sorafenib in the SHARP study in a Child-Pugh A population of patients. Indeed, a subgroup analysis from the GIDEON study that stratified patients with Child-Pugh B status according to individual scores (B7, B8 and B9) showed that median OS shortened as the Child-Pugh B score increased in severity [10].

\section{Using sorafenib in the elderly}

Trials of sorafenib in elderly patients are scarce, and to our knowledge, only three studies - all cohort studies - have evaluated treatment with sorafenib in this patient population. The efficacy results varied among these studies, with reported median survival values ranging from 5.3 to 16 months. With regards to safety profiles, certain AEs were observed more frequently, as may be expected in an elderly population that is inherently more likely than younger patients to have comorbidities that may affect tolerability.

In the only prospective cohort study, Di Costanzo and colleagues compared treatment with sorafenib at $800 \mathrm{mg} /$ day in 90 younger patients (age $<70$ years) and 60 older patients (age $>70$ years) with compensated cirrhosis and advanced HCC or HCC that was not eligible for locoregional therapies [57]. Both TTP and OS were shorter in the younger group compared with the older patients ( 8 vs 12 months and 12 vs 16 months, respectively). Although the general safety profile was similar, grade 3 and 4 AEs were observed more frequently in the younger than the older group (15.7 vs 9.2\%).

Of the two other retrospective trials, only one was comparative. It included 172 consecutive patients with advanced HCC and compensated cirrhosis treated with sorafenib and reported that OS (5.32 vs 5.16 months) and AEs related to sorafenib were comparable in a cohort of 35 elderly patients ( $\geq 70$ years) and one of 135 younger patients ( $<70$ years). Unsurprisingly, the older patients more frequently experienced comorbid cardiovascular conditions, but were also found to suffer grade 3 or 4 malaise, mucositis and neutropenia significantly more frequently than the younger cohort [58].

In a single-arm study, 60 elderly patients (>70 years) with advanced HCC were started on a low dose of sorafenib $(400 \mathrm{mg} /$ day $)$ that was scaled up to $600 \mathrm{mg} /$ day after 2 weeks and then $800 \mathrm{mg} /$ day after another 2 weeks, providing no AEs or impairment of residual liver function occurred. Ultimately, $18.3 \%$ of these patients were able to reach the full daily dose of sorafenib. The median OS in this study was 10.0 months [59].

Finally, the results of a multivariate analysis from the SOFIA field study showed that age was not significantly associated with mortality, but it was independently related to discontinuation of therapy due to intolerance [9].

\section{Treatment beyond progression}

For patients who experience progression during sorafenib treatment, further treatment options are limited. According to a consensus statement of European HCC specialists, the first option for these patients is inclusion in second-line clinical trials where available [60].

Should alternative therapies with proven efficacy in the second-line setting not be available, the consensus statement recommends the continuation of sorafenib treatment after disease progression, which may be beneficial in slowing down tumor growth [60-62]. Evidence supporting this approach has recently come 
from a study measuring the size of metastatic lesions in patients with advanced HCC following their first radiologic progression of disease while receiving sorafenib [62]. The patients were divided into those either continuing to receive sorafenib $(\mathrm{n}=23)$ or those stopping treatment (n $=13$ ). There was no increase in the growth rate of lesions after progression in patients who continued sorafenib treatment, while the growth rate was seen to increase in those patients who had stopped sorafenib after progression $(\mathrm{p}=0.002)$. Survival beyond first progression was also longer in the postprogression sorafenib continuers (median: 11.9 months) than in the discontinuers (median: 5.2 months; $\mathrm{p}=0.012$ ) [62]. However, as the postprogression survival of patients continuing sorafenib exceeded the median OS of all patients in the SHARP trial, as well as most other sorafenib studies in HCC, this study may be subject to selection bias that limits the ability to interpret the results.

In a study by Rimassa et al., the strategy of sorafenib dose escalation upon disease progression while on sorafenib failed to demonstrate any improvement in clinical outcome [63]. This prospective study did not meet its primary end point of improved PFS in patients who were escalated to sorafenib $1200 \mathrm{mg} /$ day $(\mathrm{n}=49)$ compared with those who received best supportive care ( $\mathrm{n}=$ 52) following radiologic disease progression while on sorafenib $800 \mathrm{mg} /$ day; the PFS values were 3.91 vs 2.69 months, respectively $(p=0.086)$.

Expert opinion thus concludes that sorafenib may be continued after disease progression for patients with stable performance status, although there is currently no clear evidence from large studies supporting the effectiveness of this approach $[60,61]$.

\section{Conclusion}

HCC is extremely heterogeneous in its nature and in most cases develops on a background of liver cirrhosis that has its own natural history. Therefore, demonstration of effectiveness of therapies in this disease is a complex and difficult task. To date, sorafenib is the only available systemic therapy for patients with more advanced stages of HCC and as such needs to be used effectively in daily clinical practice in order to maximize patient outcomes. For one, such effective use can be achieved by a clear understanding of how to manage AEs through sorafenib dose modification and effective prophylactic measures, which are critical for maintaining patient adherence and thus extending treatment duration. Furthermore, employing the appropriate criteria for assessing tumor responses to sorafenib in order to prevent premature treatment discontinuation is also enormously important.

While there are currently no reliable biomarkers of response for guiding treatment decisions with sorafenib, this represents an important area of ongoing research that will help to further refine our lessons with this valuable agent in the future. The data available for sorafenib in Child-Pugh $\mathrm{B}$ patients suggest the potential feasibility of this treatment in this population. However, more robust studies are necessary before confirming the use of sorafenib in this subset of patients and results from ongoing studies are eagerly awaited. For the elderly population of patients with HCC, treatment with sorafenib is feasible, although their potentially higher comorbidity status needs to be taken into consideration.

There are currently no approved second-line options for patients who progress on sorafenib. Patients should be recruited into second-line trials where available. Sorafenib may be continued after disease progression, but additional data are required in order to confirm the effectiveness of this approach.

\section{Future perspective}

Sorafenib will continue to be a valuable firstline treatment option for patients with advanced HCC or intermediate-stage patients who are unsuitable for or refractory to treatment with TACE. The use of sorafenib will be further optimized in the future through continued physician and patient education, and eventually by reliable biomarker analyses coming online. Despite recent clinical trials in the first- and second-line setting that have failed to reach their clinical end points, we must have hope that new systemic agents will be approved in the future in order to further improve clinical outcomes in patients with HCC. Once this is realized, the question of the appropriate use of sorafenib in relation to other treatments, be it in sequence or in combination, will undoubtedly be the subject of much debate and clinical investigation.

Financial \& competing interests disclosure

$L$ Bolondi has received consulting and lecture fees from Bayer HealthCare Pharmaceuticals, BMS, Bracco, Syrtex. A Craxi has attended advisory boards for Bayer. F Trevisani 
has received grant and research support from Bayer. $B$ Daniele has attended advisory boards and conferences for Bayer. S Fagiuoli has attended advisory boards and/or speaker bureaus for BMS, Gilead, MSD, Bayer, Janssen, Roche, AbbVie, Novartis, Grifols, Biotest and Kedrion. $P$ Bruzzi has received consulting and lecture fees and/or attended advisory boards for Novartis, Roche, BMS, Bayer and Merck Serono. F Spandonaro has received consulting and lecture fees from Bayer HealthCare Pharmaceuticals. $C$ Boni has attended advisory boards for Bayer, Roche, Sanofi and Celgene. A Santoro has attended advisory boards and conferences for Bayer. $M$ Colombo has received grant and research support from BMS and Gilead Science, has served on advisory committees for Merck, Roche, Novartis, Bayer, BMS, Gilead Science, Tibotec, Vertex, Janssen Cilag, Achillion, Lundbeck, GSK, GenSpera, AbbVie, AlfaWasserman and Jennerex and has received lecture fees from Tibotec, Roche, Novartis, Bayer, BMS, Gilead Science, Vertex, Merck, Janssen and Sanofi. The authors have no other relevant affliations or financial involvement with any organization or entity with a financial interest in or financial conflict with the subject matter or materials discussed in the manuscript apart from those disclosed.

The authors are grateful to 7.4 Ltd for providing medical writing assistance on this manuscript, supported by Bayer $S p A$.

\section{EXECUTIVE SUMMARY}

\section{The importance of starting right: sorafenib should be initiated at the approved dose}

- Sorafenib is the only available systemic therapy that is recommended for patients with advanced hepatocellular carcinoma and those who are not suitable for transarterial chemoembolization or have transarterial chemoembolization-refractory disease. Treatment with sorafenib should be initiated at the recommended dose of $800 \mathrm{mg} /$ day as there is no evidence to suggest that starting at a lower dose ensures the same clinical outcomes or improves tolerability. In particularly fragile patients (e.g., Child-Pugh B status, elderly and/or clinically significant cardiovascular comorbidity), a 1-month ramp-up strategy may be acceptable in order to test individual tolerability.

\section{To stop or not to stop sorafenib? When is the question}

- Having a clear understanding of how to manage adverse events (AEs) through sorafenib dose modification and effective prophylactic measures is critical for maintaining patient adherence and extending treatment duration. Rather than discontinuing sorafenib too early at the appearance of the first $A E$, sorafenib should be continued through dose modification and effective $A E$ management strategies in order to maximize patient outcomes.

- Using the appropriate criteria for assessing tumor responses to sorafenib is also important for preventing premature discontinuation. The modified Response Evaluation Criteria In Solid Tumors more accurately measures tumor responses compared with the Response Evaluation Criteria In Solid Tumors. Preliminary data suggest that the modified Response Evaluation Criteria In Solid Tumors could be used in order to assess response to sorafenib, but confirmation in larger, prospective studies is required.

- Sorafenib should not be discontinued on radiologic response alone, but patients should also be assessed for other outcomes, such as symptomatic progression, in order to extend overall survival and quality of life.

- While there are currently no reliable biomarkers of response for guiding treatment decisions with sorafenib, this represents an important area of ongoing research that will help to further refine our lessons with this valuable agent in the future.

\section{Using sorafenib in Child-Pugh B patients}

- Data available for sorafenib in Child-Pugh B patients suggest the potential feasibility of this treatment in this population. However, more robust studies are necessary before confirming the use of sorafenib in this subset of patients, and results from ongoing studies are awaited.

\section{Using sorafenib in the elderly}

- The data available for sorafenib in elderly patients suggest the potential feasibility of this treatment in this population, bearing in mind that comorbidities are likely to be more frequent.

\section{Treatment beyond progression}

- There are currently no approved second-line options for patients who progress on sorafenib. Patients should be recruited into second-line trials where available. Sorafenib may be continued after disease progression, but additional data are required in order to confirm the effectiveness of this approach. 


\section{References}

Papers of special note have been highlighted as:

- of interest; $\bullet$ of considerable interest

1 Nishida N, Kudo M. Recent advancements in comprehensive genetic analyses for human hepatocellular carcinoma. Oncology 84(Suppl. 1), 93-97 (2013).

2 Cabibbo G, Enea M, Attanasio M et al. A meta-analysis of survival rates of untreated patients in randomized clinical trials of hepatocellular carcinoma. Hepatology 51(4), 1274-1283 (2010).

3 Di Marco V, De Vita F, Koskinas J et al. Sorafenib: from literature to clinical practice. Ann. Oncol. 24(Suppl. 2), ii30-ii37 (2013).

- Useful review of the clinical efficacy and safety data of sorafenib in hepatocellular carcinoma (HCC).

4 European Association for the Study of the Liver, European Organisation for Research and Treatment of Cancer. EASL-EORTC clinical practice guidelines: management of hepatocellular carcinoma. J. Hepatol. 56(4), 908-943 (2012).

- Recent guidelines for the management of HCC, including recommendations on the use of sorafenib.

5 Cheng AL, Kang YK, Chen Z et al. Efficacy and safety of sorafenib in patients in the AsiaPacific region with advanced hepatocellular carcinoma: a Phase III randomised, double-blind, placebo-controlled trial. Lancet Oncol. 10(1), 25-34 (2009).

-. Landmark trial conducted in the AsiaPacific region that led to the approval of sorafenib treatment for patients with advanced HCC.

6 Llovet JM, Ricci S, Mazzaferro V et al. Sorafenib in advanced hepatocellular carcinoma. N. Engl. J. Med. 359(4), 378-390 (2008).

-. Landmark trial leading to the approval of sorafenib treatment for patients with advanced HCC.

7 Cammà C, Cabibbo G, Petta $\mathrm{S}$ et al. Cost-effectiveness of sorafenib treatment in field practice for patients with hepatocellular carcinoma. Hepatology 57(3), 1046-1054 (2013).

8 Lencioni R, Marrero J, Venook A et al. Design and rationale for the non-interventional Global Investigation of Therapeutic DEcisions in Hepatocellular Carcinoma and Of its Treatment with Sorafenib (GIDEON) study. Int. J. Clin. Pract. 64(8), 1034-1041 (2010).

9 Iavarone M, Cabibbo G, Piscaglia F et al. Field-practice study of sorafenib therapy for hepatocellular carcinoma: a prospective multicenter study in Italy. Hepatology 54(6), 2055-2063 (2011).

- Important Italy-based study of sorafenib in the real-life setting.

10 Marrero JA, Lencioni R, Ye S-L et al. Final analysis of GIDEON (Global Investigation of Therapeutic Decisions in Hepatocellular Carcinoma [HCC] and of Its Treatment with Sorafenib [Sor]) in $>3000$ Sor-treated patients (pts): clinical findings in pts with liver dysfunction. J. Clin. Oncol. 31(Suppl.), 4126 (2013).

-. Largest study ever conducted on the use of sorafenib in the real-life setting.

11 Lencioni R, Kudo M, Ye SL et al. GIDEON (Global Investigation of therapeutic DEcisions in hepatocellular carcinoma and Of its treatment with sorafeNib): second interim analysis. Int. J. Clin. Pract. 68(5), 609-617 (2014)

12 Reig M, Rimola J, Torres F et al. Postprogression survival of patients with advanced hepatocellular carcinoma. Rationale for second line trial design. Hepatology 58(6), 2023-2031 (2013).

13 Di Costanzo GG, Tortora R, Iodice L et al. Safety and effectiveness of sorafenib in patients with hepatocellular carcinoma in clinical practice. Dig. Liver Dis. 44(9), 788-792 (2012).

14 Pressiani T, Boni C, Rimassa L et al. Sorafenib in patients with Child-Pugh class A and $\mathrm{B}$ advanced hepatocellular carcinoma: a prospective feasibility analysis. Ann. Oncol. 24(2), 406-411 (2013).

15 Shingina A, Hashim AM, Haque M et al. In a 'real-world', clinic-based community setting, sorafenib dose of $400 \mathrm{mg} /$ day is as effective as standard dose of $800 \mathrm{mg} /$ day in patients with advanced hepatocellular carcinoma, with better tolerance and similar survival. Can. J. Gastroenterol. 27(7), 393-396 (2013).

16 Cainap C, Qin S, Huang W-T et al. Phase III trial of linifanib versus sorafenib in patients with advanced hepatocellular carcinoma (HCC). J. Clin. Oncol. 31(Suppl.), 249 (2013).

17 Cheng AL, Kang YK, Lin DY et al. Sunitinib versus sorafenib in advanced hepatocellular cancer: results of a randomized Phase III trial. J. Clin. Oncol. 31(32), 4067-4075 (2013).

18 Johnson PJ, Qin S, Park JW et al. Brivanib versus sorafenib as first-line therapy in patients with unresectable, advanced hepatocellular carcinoma: results from the randomized Phase III BRISK-FL study. J. Clin. Oncol. 31(28), 3517-3524 (2013).
19 Zhu AX, Rosmorduc O, Evans J et al. SEARCH: a Phase III, randomized, double-blind, placebo-controlled trial of sorafenib plus erlotinib in patients with hepatocellular carcinoma (HCC). Ann. Oncol. 23(Suppl. 9), LBA2 (2012).

20 Abou-Alfa GK, Schwartz L, Ricci S et al. Phase II study of sorafenib in patients with advanced hepatocellular carcinoma. J. Clin. Oncol. 24(26), 4293-4300 (2006).

21 Strumberg D, Richly H, Hilger RA et al. Phase I clinical and pharmacokinetic study of the novel Raf kinase and vascular endothelial growth factor receptor inhibitor BAY 43-9006 in patients with advanced refractory solid tumors. J. Clin. Oncol. 23(5), 965-972 (2005).

22 Marrero JA, Venook A, Kudo M et al. Second interim analysis of GIDEON (Global Investigation of therapeutic DEcisions in unresectable hepatocellular carcinoma and of its treatment with sorafeNib): subgroup analysis by initial sorafenib dose. Hepatology 54(Suppl. 4), 2192 (2011).

23 Daniele B, Croitoru AE, Papandreou C et al. Analysis of sorafenib starting dose and outcomes from the European subset of GIDEON (Global Investigation of Therapeutic Decisions in Hepatocellular Carcinoma and of its Treatment with Sorafenib [SOR]) in $>1000$ patients (pts). Eur. J. Cancer 49 (Suppl. 2), 2581 (2013).

- Interesting analysis of the starting dose effect with sorafenib.

24 Verslype C, Rosmorduc O, Rougier P et al. Hepatocellular carcinoma: ESMO-ESDO Clinical Practice Guidelines for diagnosis, treatment and follow-up. Ann. Oncol. 23(Suppl. 7), vii41-vii48 (2012).

- Recent guidelines for the management of HCC, including recommendations on the use of sorafenib.

25 Abou-Alfa GK. Sorafenib use in hepatocellular carcinoma: more questions than answers. Hepatology 60 (1), 15-18 (2014).

26 Vincenzi B, Santini D, Russo A et al. Early skin toxicity as a predictive factor for tumor control in hepatocellular carcinoma patients treated with sorafenib. Oncologist 15(1), 85-92 (2010).

27 Reig M, Torres F, Rodriguez-Lope C et al. Early dermatologic adverse events predict better outcome in HCC patients treated with sorafenib. J. Hepatol. 61(2), 318-324 (2014).

28 Hatzivassiliou G, Song K, Yen I et al. RAF inhibitors prime wild-type RAF to activate 
the MAPK pathway and enhance growth. Nature 464(7287), 431-435 (2010).

29 Spirli C, Morell CM, Locatelli L et al. Cyclic AMP/PKA-dependent paradoxical activation of Raf/MEK/ERK signaling in polycystin-2 defective mice treated with sorafenib. Hepatology 56(6), 2363-2374 (2012).

30 Miller AA, Murry DJ, Owzar K et al. Phase I and pharmacokinetic study of sorafenib in patients with hepatic or renal dysfunction: CALGB 60301. J. Clin. Oncol. 27(11), 1800-1805 (2009).

31 Abou-Alfa GK, Schwartz L, Ricci S et al. Phase II study of sorafenib in patients with advanced hepatocellular carcinoma. J. Clin. Oncol. 24(26), 4293-4300 (2006).

32 Bolondi L, Cillo U, Colombo $\mathrm{M}$ et al. Position paper of the Italian Association for the Study of the Liver (AISF): the multidisciplinary clinical approach to hepatocellular carcinoma. Dig. Liver Dis. 45(9), 712-723 (2013).

33 Abbadessa G, Rimassa L, Pressiani T et al. Optimized management of advanced hepatocellular carcinoma: four long-lasting responses to sorafenib. World J. Gastroenterol. 17(19), 2450-2453 (2011).

34 Cabibbo G, Maida M, Camma C et al. Is the efficacy of sorafenib treatment in patients with hepatocellular carcinoma affected by age? Expert Rev. Anticancer Ther. 13(12), 1355-1361 (2013).

35 Bracarda S, Ruggeri EM, Monti M et al. Early detection, prevention and management of cutaneous adverse events due to sorafenib: recommendations from the Sorafenib Working Group. Crit. Rev. Oncol. Hematol. 82(3), 378-386 (2012).

36 Cabibbo G, Rolle E, De Giorgio M et al. Management of cirrhotic patients with hepatocellular carcinoma treated with sorafenib. Expert Rev. Anticancer Ther. 11(12), 1807-1816 (2011).

37 D'Angelo S, Secondulfo M, De Cristofano R et al. Selection and management of hepatocellular carcinoma patients with sorafenib: recommendations and opinions from an Italian liver unit. Future Oncol. 9(4), 485-491 (2013).

38 Edmonds K, Hull D, Spencer-Shaw A et al. Strategies for assessing and managing the adverse events of sorafenib and other targeted therapies in the treatment of renal cell and hepatocellular carcinoma: recommendations from a European nursing task group. Eur. J. Oncol. Nurs. 16(2), 172-184 (2012).

39 Scandurra G, Aiello RA, Ali M et al. Appropriate management of cutaneous adverse events maximizes compliance with sorafenib treatment: a single-center experience. Future Oncol. 8(5), 609-615 (2012).

40 Schott E, Ebert MP, Trojan J. Treatment of hepatocellular carcinoma with sorafenib focus on special populations and adverse event management. Z. Gastroenterol. 50(9), 1018-1027 (2012).

41 Wood LS, Lemont H, Jatoi A et al. Practical considerations in the management of hand-foot skin reaction caused by multikinase inhibitors. Commun. Oncol. 7 , 23-29 (2010).

42 Lencioni R, Llovet JM. Modified RECIST (mRECIST) assessment for hepatocellular carcinoma. Semin. Liver Dis. 30(1), 52-60 (2010).

43 Eisen T, Sternberg CN, Robert C et al. Targeted therapies for renal cell carcinoma: review of adverse event management strategies. J. Natl Cancer Inst. 104(2), 93-113 (2012).

44 Shinohara N, Nonomura N, Eto M et al. A randomized multicenter Phase II trial on the efficacy of a hydrocolloid dressing containing ceramide with a low-friction external surface for hand-foot skin reaction caused by sorafenib in patients with renal cell carcinoma. Ann. Oncol. 25(2), 472-476 (2014).

45 Bellmunt J, Eisen T, Fishman M et al. Experience with sorafenib and adverse event management. Crit. Rev. Oncol. Hematol. 78(1), 24-32 (2011).

46 Campos MP, Hassan BJ, Riechelmann R et al. Cancer-related fatigue: a practical review. Ann. Oncol. 22(6), 1273-1279 (2011).

47 Colombo M, Raoul JL, Lencioni R et al. Multidisciplinary strategies to improve treatment outcomes in hepatocellular carcinoma: a European perspective. Eur. J. Gastroenterol. Hepatol. 25(6), 639-651 (2013).

48 Therasse P, Arbuck SG, Eisenhauer EA et al. New guidelines to evaluate the response to treatment in solid tumors. European Organization for Research and Treatment of Cancer, National Cancer Institute of the United States, National Cancer Institute of Canada. J. Natl Cancer Inst. 92(3), 205-216 (2000).

49 Kawaoka T, Aikata H, Murakami E et al. Evaluation of the mRECIST and alphafetoprotein ratio for stratification of the prognosis of advanced-hepatocellularcarcinoma patients treated with sorafenib. Oncology 83(4), 192-200 (2012).
50 Edeline J, Boucher E, Rolland Y et al. Comparison of tumor response by response evaluation criteria in solid tumors (RECIST) and modified RECIST in patients treated with sorafenib for hepatocellular carcinoma. Cancer 118(1), 147-156 (2012).

51 Llovet JM, Pena CE, Lathia CD et al. Plasma biomarkers as predictors of outcome in patients with advanced hepatocellular carcinoma. Clin. Cancer Res. 18(8), 2290-2300 (2012).

52 Personeni N, Bozzarelli S, Pressiani T et al. Usefulness of alpha-fetoprotein response in patients treated with sorafenib for advanced hepatocellular carcinoma. J. Hepatol. 57(1), 101-107 (2012).

53 Nakazawa T, Hidaka H, Takada J et al. Early increase in alpha-fetoprotein for predicting unfavorable clinical outcomes in patients with advanced hepatocellular carcinoma treated with sorafenib. Eur. J. Gastroenterol. Hepatol. 25(6), 683-689 (2013).

54 Kuzuya T, Asahina Y, Tsuchiya K et al. Early decrease in alpha-fetoprotein, but not des-gamma-carboxy prothrombin, predicts sorafenib efficacy in patients with advanced hepatocellular carcinoma. Oncology 81(3-4), 251-258 (2011).

55 Shao YY, Lin ZZ, Hsu C et al. Early alpha-fetoprotein response predicts treatment efficacy of antiangiogenic systemic therapy in patients with advanced hepatocellular carcinoma. Cancer 116(19), 4590-4596 (2010).

56 Estfan B, Byrne M, Kim R. Sorafenib in advanced hepatocellular carcinoma: hypertension as a potential surrogate marker for efficacy. Am. J. Clin. Oncol. 36(4), 319-324 (2013).

57 Di Costanzo GG, Tortora R, De LM et al. Impact of age on toxicity and efficacy of sorafenib-targeted therapy in cirrhotic patients with hepatocellular carcinoma. Med. Oncol. 30 (1), 446 (2013).

58 Wong H, Tang YF, Yao TJ et al. The outcomes and safety of single-agent sorafenib in the treatment of elderly patients with advanced hepatocellular carcinoma (HCC). Oncologist 16(12), 1721-1728 (2011).

59 Montella L, Addeo R, Cennamo G et al. Sorafenib in elderly patients with advanced hepatocellular carcinoma: a case series. Oncology 84(5), 265-272 (2013).

60 Peck-Radosavljevic M, Greten TF, Lammer $\mathrm{J}$ et al. Consensus on the current use of sorafenib for the treatment of hepatocellular carcinoma. Eur. J. 
Gastroenterol. Hepatol. 22(4), 391-398 (2010).

61 He AR, Goldenberg AS. Treating hepatocellular carcinoma progression following first-line sorafenib: therapeutic options and clinical observations. Ther. Adv. Gastroenterol. 6(6), 447-458 (2013).
62 Miyahara K, Nouso K, Morimoto Y et al. Efficacy of sorafenib beyond first progression in patients with metastatic hepatocellular carcinoma. Hepatol. Res. 44(3), 296-301 (2014).

63 Rimassa L, Pressiani T, Boni C et al. A Phase II randomized dose escalation trial of sorafenib in patients with advanced hepatocellular carcinoma. Oncologist 18(4), 379-380 (2013).

64 National Comprehensive Cancer Network. NCCN clinical practice guidelines in oncology: cancer-related fatigue.

www.nccn.org 